\title{
VARIABLES MACROECONÓMICAS Y MICROECONÓMICAS QUE INFLUYEN EN LA ESTIMACIÓN DEL COSTO DE CAPITAL: UN ESTUDIO DE CASO*
}

\author{
DANIEL CARDONA ECHEVERRI", SANDRA GAITÁN RIAÑO"*** \& HERMILSON VELÁSQUEZ CEBALLOS \\ UNIVERSIDAD EAFIT
}

Recibido/ Received/ Recebido: 13/11/2015-Aceptado/ Accepted / Aprovado: 28/06/2016

\section{Resumen}

\begin{abstract}
Este artículo identifica y contrasta la significancia estadística de variables macroeconómicas y microeconómicas, que desde la teoría pueden influir sobre el costo de capital. Utilizando información de las empresas listadas en la Bolsa de Valores de Colombia (BVC) entre los periodos 2003-4 a 2012-1 y modelos de paneles dinámicos para explicar el Costo de Capital Promedio Ponderado (WACC), se encuentra que la mayoría de las variables propuestas resultan estadísticamente significativas, así mismo se prueba la existencia de inercia entre el costo de capital actual y el del periodo pasado.

Palabras clave: Costo de capital; WACC; Variables macroeconómicas; Variables microeconómicas.

\section{MACRO-AND MICROECONOMIC VARIABLES AFFECTING THE ESTIMATED COST OF CAPITAL: A CASE STUDY}

\begin{abstract}
This article identifies and contrasts the statistical significance of macroeconomic and microeconomic variables, which can influence the cost of capital from theory. Using information from companies listed on the Colombia Stock Exchange (BVC) between the periods 2003-4 and 2012-1 and dynamic panel models to explain the Weighted Average Cost of Capital (WACC). It is found that most proposed variables are statistically significant. It also proves the existence of inertia between the current cost of capital and the last period.

Keywords: Capital cost; WACC; Macroeconomic variables; Microeconomic variables.
\end{abstract}

Artículo de investigación derivado del trabajo de grado titulado "El costo del uso del capital. Un modelo econométrico" de la Maestría en Administración Financiera de la Universidad EAFIT.

- Master en Administración Financiera (Universidad EAFIT, Colombia) y en Estrategia y Emprendimiento (Universidad de Montpellier 1, Francia). Correo electrónico: cardona.daniel03@gmail.com

... Profesor Escuela de Economía y Finanzas, Universidad EAFIT. AA 3300 Medellín (Colombia). Dirección postal: Carrera 49 N 7 sur - 50, Medellín - Colombia. Teléfono: (+574)2619500 Ext 9585. Correo electrónico: sgaitanr@eafit.edu.co

.... Profesor Escuela de Economía y Finanzas, Universidad EAFIT. AA 3300 Medellín (Colombia). Correo electrónico: evelas@eafit.edu.co 


\title{
VARIÁVEIS MACROECONÔMICAS E MICROECONÔMICAS QUE INFLUENCIAM A ESTIMATIVA DO CUSTO DE CAPITAL: UM ESTUDO DE CASO
}

\begin{abstract}
Resumo
Este artigo identifica e contrasta a significância estatística de variáveis macroeconômicas e microeconômicas, que desde a teoria podem influenciar o custo de capital. Utilizando informação das empresas listadas na Bolsa de Valores de Colômbia (BVC) entre os períodos 2003-4 a 2012-1 e modelos de painéis dinâmicos para explicar o Custo de Capital Médio Ponderado (WACC), encontra-se que a maioria das variáveis propostas resultam estatisticamente significativas, como também se prova a existência de inércia entre o custo de capital atual e o do período passado.

Palavras chave: Custo de capital; WACC; Variáveis macroeconómicas; Variáveis microeconómicas.
\end{abstract}

Cardona, D., Gaitán, S. \& Velásquez, H. (2017). Variables macroeconómicas y microeconómicas que influyen en la estimación del costo de capital: un estudio de caso. En: Revista de la Facultad de Ciencias Económica: Investigación y Reflexión. rev.fac.cienc.econ, XXV(1). DOI: http://dx.doi.org/10.18359/rfce.2657

JEL: C33, G32.

\section{Introducción}

En los últimos años Colombia se ha convertido en destino para inversionistas extranjeros y las empresas han desarrollado estrategias para lograr su inserción en el mercado internacional, lo cual se ve favorecido por el diseño de políticas gubernamentales que promueven la realización de nuevos tratados de libre comercio. Lo anterior refleja la necesidad de realizar investigaciones que permitan al inversionista disponer de elementos de juicio para hacer una valoración científica sobre los posibles resultados que se pueden presentar al realizar una inversión en el mercado Colombiano, así como también suministrar a los agentes información de cuan sensible es el costo de capital a choques en variables económicas que pueden afectar directamente a las firmas.

Gran parte de las investigaciones se han centrado en establecer los determinantes macroeconómicos y microeconómicos para la estructura de capital, sin embargo sobre el costo de capital, variable funda- mental en los análisis de inversión, son pocos los estudios realizados especialmente en América Latina.

El teorema de Modigliani \& Miller (1958, 1961, 1963) comprendido en una serie de papers, plantea la base de las finanzas corporativas modernas. Bajo el supuesto de mercados perfectos el teorema establece en su preposición I que el valor de la empresa no se ve afectado por las decisiones de financiación. Por su parte, la preposición II establece que el apalancamiento no afecta el costo promedio ponderado de capital. La preposición III establece que el valor de mercado de la empresa es independiente de su política de dividendos. Y la preposición IV plantea que los tenedores de capital son indiferentes a la política de financiación.

A partir de estos planteamientos se han desarrollado diferentes teorías acerca de la estructura de capital, por ejemplo, Kraus \& Litzenberger (1973) 
en su teoría del "trade-off" establecen la existencia de una estructura óptima de capital en la cual los costos marginales se igualan a los beneficios marginales. Bacter (1967), Stiglitz (1969), Kraus \& Litzemberger (1973), Kim (1978) y Altman (1984) citados por Rivera (2002) en la teoría de bancarrota, consideran que a medida que la empresa se endeuda existe mayor riesgo de quiebra. Jensen \& Meckling (1976), Jensen (1986), Williamson (1988), Stulz (1990), Harris \& Raviv (1991), Myers (1977), Hirshleifer \& Thakor (1992) y Diamond (1989) con la teoría de los costos de agencia, argumentan que existen unos costos asociados por la separación entre la propiedad y el control. Myers \& Majluf (1984) con la teoría de jerarquía en las fuentes de financiación "Peking order theory" argumentan que las empresas prefieren utilizar recursos propios y en caso de requerir financiación prefieren deuda.

Autores como Fuenzalida \& Mongrut (2010) realizan una comparación de los principales métodos para estimar el costo del capital propio en mercados emergentes para luego aplicarlos a América Latina. En ese estudio, los autores encuentran disparidad en los resultados y dificultades para implementar las metodologías de estimación de la tasa.

Sin embargo, para el caso de estudios con variables macroeconómicas y microeconómicas, autores como Levy (2000); Hackbarth, Miao \& Morellec (2006); Bokpin (2009); Camara (2012); y Pozzo (2005) realizan diferentes estudios donde consideran estas variables para estimar los determinates de la estructura de capital.

En las finanzas, es muy utilizado el costo promedio ponderado del capital WACC como el costo de capital para la toma de decisiones en los proyectos de inversión. Sin embargo, Ang (1973) y Lewellen (1974) han planteado la necesidad de estudiar la forma cómo se estima el costo promedio ponderado del capital WACC, algunos autores consideran válida su estimación cuando se hace como el costo promedio de los retornos esperados de los títulos, para el caso de firmas individuales donde la estructura de capital y la tasa de pago de la deuda son conocidas. Sin embargo en el caso corporativo donde hay presencia de deuda privada como una forma de financiamiento, el costo de capital es difícil de estimar (Fama \& French, 1999).

Las consideraciones anteriores sobre el costo de capital WACC hacen que sea pertinente tratar de explicar sus variaciones a través de variables macroeconómicas y microeconómicas, enfoque que tiene pocos estudios a nivel mundial, muy escasos a nivel latinoamericano y en Colombia no hay registros de estudios sobre este tema con esta perspectiva.

El aporte central de este trabajo es identificar variables macroeconómicas y microeconómicas así como también la dinámica que permita explicar el proceso generador de los datos para el costo de capital de las empresas que participan en la construcción del índice del IGBC y así, los inversionistas puedan considerar estrategias para minimizar la incertidumbre frente a los retornos esperados.

En esta investigación se identifican y contrastan la significancia estadística de variables macroeconómicas y microeconómicas, que afectan el comportamiento del costo del capital en algunas empresas que cotizan o han cotizado en la Bolsa de Valores de Colombia (BVC), durante el periodo 2003-4 al 2012-1.

En este estudio a partir de los modelos estimados, se presenta evidencia acerca de la significancia estadística y de la coherencia en signos de las variables consideradas. Además los factores macroeconómicos como el PIB y la DTF presentan un mayor impacto en el costo de capital, en contraste con los factores microeconómicos. Estos resultados son congruentes con el planteamiento de autores como Camara (2012), Korajczyk \& Levy (2003) y Booth et al. (2001) citado por Stulz (2005), para el caso de la estructura de capital.

El resto de este trabajo está distribuido de la siguiente forma: en la sección 2 se presentan los fundamentos teóricos; la sección 3 está compuesta por la propuesta metodológica, la explicación sobre las variables a utilizar y los datos; en la sección 4 se presentan los resultados de la estimación y sus análisis; y por último, la sección 5 resume y concluye el trabajo. 


\section{Marco Teórico}

El proceso de globalización en el cuál se encuentran la mayoría de los países, hace que los mercados se vean afectados por movimientos de la economía internacional. Como es sabido, las firmas constituyen el eje central de la actividad económica en la mayoría de los países, razón por la cual es importante conocer dentro de la teoría financiera los elementos que caracterizan el costo de capital. Por esta razón analizar su comportamiento y el efecto que sobre él ejercen factores macroeconómicos y microeconómicos resulta de interés en la viabilidad y sostenibilidad de los proyectos de inversión.

Modigliani \& Miller $(1958,1961,1963)$ en su teorema afirman que, en mercados perfectos y precios justos, el costo del capital de una empresa no se ve afectado por la decisión de su estructura de capital ya que este es constante sin importar el nivel de apalancamiento que ella tenga $y$, adicionalmente, el valor de la compañía no se verá afectado al momento de ser valorada por la metodología de flujos futuros descontados.

Levy \& Hennessy (2007) estudian el conflicto que existe entre los costos de agencia y la estructura de capital de una empresa y como varía esta con el ciclo económico, la investigación concluye que la protección al inversionista es importante para la estabilidad macroeconómica, encontrando menor fragilidad en países con alta protección al inversionista. Hackbarth, Miao \& Morellec (2006), encuentran que los factores macroeconómicos afectan la decisión de apalancamiento óptima para la empresa. Bokpin (2009) realiza un estudio de datos de panel para 37 economías y encuentra que las variables macroeconómicas impactan la decisión de la estructura de capital en las empresas. Camara (2012), para empresas de Estados Unidos, encuentra que los factores y las condiciones macroeconómicas tienen un efecto significativo en la dinámica de las estructuras de capital.

En la misma línea, Stulz (2005) indica que algunos estudios encuentran que los factores a nivel país ayudan a explicar la estructura de capital. Este autor menciona que Booth et al. (2001) y Fan, Titman \&
Twite (2003) coinciden en afirmar que la estructura de capital es determinada en mayor medida por factores macroeconómicos que a nivel de firma o de industria. Además, Rajan \& Zingales (1995) encuentra que existe la misma relación entre las variables a nivel de firma de los países del G-7.

Easley \& O'hara (2004) centran su estudio en factores microeconómicos como el rol de la información privada y pública sobre el costo de capital de las firmas. Mediante un modelo de equilibrio con expectativas racionales encuentran que, un inversionista en posesión de información privada requiere de mayores rendimientos para conservar los activos, mientras que agentes con poca información están más dispuestos a mantenerlos debido a que los inversionistas informados tienen mayor capacidad de cambiar las proporciones de su portafolio, con el fin de optimizarlo considerando la nueva información. Otro importante hallazgo de su investigación es que, una firma puede afectar su costo de capital mediante la precisión y cantidad de información que disponga para los inversionistas, es decir, de la revelación que haga sobre sus estándares de contabilidad y de sus políticas corporativas.

Bellalah \& Belhaj (2003) argumentan que debido a la posible presencia de información asimétrica el costo de capital en los países pequeños es determinado globalmente y no localmente como se acostumbra a estimar (implementando CAPM con un índice local que revele el portafolio de mercado local), en presencia de información incompleta, ésta información, es definida bajo el modelo de equilibrio de mercado de capitales con información incompleta de Merton (1987), más conocido como CAPMI.

Camacho \& Meneses (2013) consideran el impacto que tiene la fijación de precios sobre el costo de capital, estos autores argumentan que esta es una variable endógena, la cual depende del precio fijado. La principal conclusión de su investigación es que el costo de capital crece en la medida en que el nivel de precios fijado disminuye.

Para Latinoamérica se puede mencionar el estudio de Pozzo (2005), quien utiliza un modelo de panel de datos para estimar el impacto de las variables 
macroeconómicas en la estructura de capital de las empresas latinoamericanas, y halla que las empresas se ven obligadas a financiarse más con recursos propios que con externos. Por su parte, para el caso Colombiano, Salazar (2009) realiza un estudio econométrico para la estructura del capital y el endeudamiento óptimo en las empresas colombianas utilizando el método generalizado de los momentos GMM, encontrando resultados acordes a la literatura. En adición, Gama (2007) analiza el efecto del costo del uso de capital y del costo promedio ponderado de capital de las Pymes del sector avícola de Bogotá en los estados financieros.

El estudio del costo de capital se aborda a partir de variables de la macroeconomía y la microeconomía. Entre estas consideramos variables como el PIB, la DTF, Q de Tobin, liquidez, la tangibilidad, el crecimiento en ventas y el margen de ganancias algunas de ellas propuesta por autores como Hackbarth et al. (2006), Pozzo (2005), Camara (2012) y Bokpin (2009), además de otras que se han considerado importantes como el rendimiento del IGBC, dado que es un indicador de referencia de rendimiento del mercado accionario Colombiano.

Para estimar el costo de capital de las empresas se sigue la metodología empleada por Upadhyay \&
Sriram (2011) quienes consideran como costo de capital el WACC, el cual está definido como el costo promedio ponderado de las diferentes fuentes de financiación y se expresa como:

$$
W A C C=\frac{D}{D+E} k_{D}+\frac{E}{D+E} k_{E}
$$

Donde:

D: Valor del mercado de la deuda

E: Valor del mercado del capital propio $\mathrm{k}_{\mathrm{D}}$ : Costo de la deuda después de impuestos $\mathrm{k}_{\mathrm{E}}$ : Costo del capital propio

Dentro de la definición de costo de capital WACC se encuentra la estructura de capital conocida como la participación de las diferentes fuentes de financiación de la empresa dentro del capital total. En la tabla 1 se presenta el planteamiento principal de las principales teorías sobre estructura de capital.

En las empresas, es de uso común el costo promedio ponderado del capital WACC como el costo de capital. Ang (1973) y Lewellen (1974) han planteado la necesidad de estudiar la forma cómo se estima el costo promedio ponderado del capital WACC, algunos autores consideran válido su cálculo cuando se hace como el costo promedio de los retornos esperados de los títulos, para el caso de firmas in-

Tabla 1. Resumen de las principales teorías sobre la estructura de capital

\begin{tabular}{|l|l|}
\hline \multicolumn{1}{|c|}{ Teoría } & \multicolumn{1}{c|}{ Principios } \\
\hline $\begin{array}{l}\text { Teoría de la irrelevancia } \\
\text { (Modigliani \& Miller, 1958,1961, 1963) }\end{array}$ & $\begin{array}{l}\text { La fuente de financiamiento no tiene importancia cuando se calcula el costo del capital debido a la } \\
\text { compensación entre el riesgo de tener deuda y la remuneración a los accionistas. }\end{array}$ \\
\hline $\begin{array}{l}\text { Teoría del Trade-off } \\
\text { (Kraus \& Litzenberger, 1973) }\end{array}$ & $\begin{array}{l}\text { Existe una estructura de capital óptima donde los costos marginales se igualan a los beneficios } \\
\text { marginales, dada la observación de los costos de bancarrota y el escudo fiscal que ofrece la } \\
\text { deuda. }\end{array}$ \\
\hline $\begin{array}{l}\text { Teoría de jerarquías en las fuentes de financia- } \\
\text { miento (Peking Order Theory) (Myers \& Majluf, } \\
\text { 1984) }\end{array}$ & $\begin{array}{l}\text { Las empresas prefieren financiarse con recursos propios y si es necesaria la búsqueda de recur- } \\
\text { sos externos tienden a preferir deuda en lugar de patrimonio. }\end{array}$ \\
\hline $\begin{array}{l}\text { Teoría de costos de bancarrota } \\
\text { (Kraus \& Litzenberger, 1973) }\end{array}$ & $\begin{array}{l}\text { Surge de la teoría del Trade-off, a medida que la empresa tiene más deuda tienen mayor riesgo de } \\
\text { quiebra. Deben ser evaluados todos los puntos que respalden la deuda. }\end{array}$ \\
\hline
\end{tabular}

Fuente: Elaboración propia. 
dividuales donde la estructura de capital y la tasa de pago de la deuda son conocidas. Sin embargo en el caso corporativo donde hay presencia de deuda privada como una forma de financiamiento, el costo de capital es difícil de estimar (Fama \& French, 1999).

Las consideraciones anteriores sobre el costo de capital WACC hacen que sea pertinente tratar de explicar sus variaciones a través de variables macroeconómicas, microeconómicas y algunos de sus rezagos, enfoque que tiene pocos estudios a nivel mundial, muy escasos a nivel latinoamericano y en Colombia no hay registros con este enfoque.

\section{Metodología}

El presente estudio utiliza una metodología cuantitativa desde un enfoque correlacional donde se busca establecer cuáles variables macroeconómicas y microeconómicas afectan el costo de capital. Para ello se utiliza la información trimestral contable y de mercado de 23 empresas que cotizan o que han cotizado en la Bolsa de Valores de Colombia (BVC) en el periodo 2003-4 y 2012-1. Se seleccionaron empresas que durante el periodo de estudio participaran del índice IGBC de la BVC, estas son: Bavaria, Cartón de Colombia, Carulla Vivero, Cementos Argos, Computec, Corferias, Ecopetrol, Enka de Colombia, Estra, ETB, Eternit Colombia, Exito, Fabricato, Gas Natural, ISA, ISAGEN, Hospital Marly, Mineros, Nutresa, ODINSA, Acerias Paz del Rio, Promigas y Tablemac. Los datos contables fueron obtenidos de Economatica, los financieros de Bloomberg y los datos macroeconómicos fueron tomados de las series estadísticas provistas por el Banco de la República de Colombia. El costo promedio ponderado de capital (WACC) fue tomado directamente de Bloomberg ${ }^{1}$.

Esta investigación se considera como variable endógena a:

\footnotetext{
Se verificó que la variable WACC fuera estimada utilizando la definición tradicional para costo promedio ponderado de capital siguiendo la definición de la ecuación (1).
}

WACC $_{i t}$ : Costo promedio ponderado del capital de la empresa i en el trimestre $t$

La forma funcional general que recoge la propuesta es:

$W_{A C C}=f$ (variables macroeconómicas, variables microeconómicas,variables de control)

La definición de la variable hace que de manera natural sea necesario considerar como variable explicativa su propio rezago y por tanto:

Las variables que se consideran como explicativas para $W_{A C C}$ son:

DPIB $_{\mathrm{t}}$ : Incremento porcentual del Producto interno bruto de Colombia para el periodo t.

DTF: Tasa de depósitos a término fijo en el tiempo t.

rentIGBC$C_{t}$ : Rentabilidad del IGBC en el tiempo t.

crecimiento $_{\mathrm{it}}$ : Es el incremento porcentual de las ventas respecto al mismo periodo del año anterior para la empresa i en el tiempo t.

liquidez $z_{\mathrm{it}}$ : Es el cociente entre activos corrientes y pasivos corrientes de la empresa i en el tiempo t.

margen_ganancias it $_{\text {: }}$ Es el cociente entre las ganancias operacionales y los costos operacionales de la empresa i en el periodo $t$.

tangibilidad $_{\mathrm{it}}$ :Es el ratio entre activos fijos netos y activos totales de la empresa i en el periodo t.

qtobin $_{\mathrm{it}}$ : Es el ratio del valor del mercado dividido por el valor de los activos de la firma i en el periodo $t$.

La forma funcional propuesta para la estimación es:

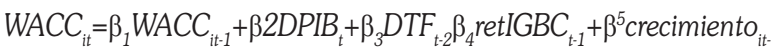
${ }_{1}+\beta_{6}$ liquidez $_{i t}+\beta_{7}$ margenganancias $_{i t}+\beta_{8}$ tangibilidad $_{i t-1}+\beta_{9}$ qtobin $_{i t}+\mu_{i t}$ 


\subsection{Variables macroeconómicas y signos esperados}

\subsubsection{Crecimiento de la economía (DPIB)}

Es el incremento porcentual del PIB respecto al mismo periodo del año anterior. El signo esperado de esta variable depende del ciclo económico en el cual se encuentre la economía. Cuando las economías se encuentran en un periodo expansionista las tasas de interés también se encontraran en aumento, de acuerdo con la Teoría de Jerarquías, en los periodos de crecimiento la firma no estará en capacidad de soportar dicho crecimiento con fondos propios así que necesitará recurrir a la deuda, debido a las altas tasas presentes y por tanto el costo de capital aumentará, en la fase recesiva se espera una disminución. Para el periodo de análisis se puede afirmar que la economía se encontraba en un periodo de crecimiento y por tanto es de esperar que $\beta_{2}>0$.

\subsubsection{DTF}

"Es el promedio ponderado de las tasas de interés efectivas de captación a 90 días (las tasas de los certificados de depósito a término (CDT) a 90 días) de los establecimientos bancarios, corporaciones financieras, compañías de financiamiento comercial y corporaciones de ahorro y vivienda. Esta tasa es calculada semanalmente por el Banco de la República" (Banco de la República, 2012). Esta variable es una proxy del movimiento del costo de la deuda de las empresas, o también se puede interpretar como una tasa alterna de inversión en renta fija. Se espera que $\beta_{3}>0$, ya que un aumento en esta tasa producirá un aumento en el costo de la deuda de la empresa y por lo tanto en el costo del capital.

\subsubsection{Rentabilidad del IGBC}

Es el resultado de calcular los retornos del Índice General de la Bolsa de Valores de Colombia (IGBC) frente al mismo trimestre del año anterior. Esta variable, considerada como una variable financiera, se interpreta como los rendimientos del mercado accionario de la economía colombiana. Es de esperar que la relación sea positiva $\left(\beta_{4}>0\right)$ en tanto que esta tasa refleja los retornos mínimos esperados de invertir en todo el mercado accionario, convirtiéndose en tasa base para calcular el costo del capital propio para la empresa mediante el modelo CAPM.

\subsection{Variables microeconómicas y signos esperados}

\subsubsection{Crecimiento}

Es el incremento porcentual de las ventas respecto al mismo periodo del año anterior. Se espera que $\beta_{5}<0$ respaldándose en la teoría del trade-off, la cual habla acerca de un menor costo de la deuda para las empresas con mayores posibilidades de crecimiento.

\subsubsection{Liquidez}

Es el cociente entre activos corrientes y pasivos corrientes. El signo que se espera es negativo $\left(\beta_{6}<0\right)$, debido a que la empresa al presentar mayor liquidez dará señales al mercado de solidez y mejor respuesta ante shocks negativos, lo que se traducirá en una reducción en las tasas mínimas de retorno (Pozzo, 2005). Sin embargo también puede ocurrir lo contrario $\left(\beta_{6}>0\right)$, debido a que si la empresa muestra excesos de liquidez el mercado la castiga por el hecho de mantener fondos ociosos.

\subsubsection{Margen de ganancias}

Se expresa como el crecimiento del margen de ganancias, el cual a su vez está definido como la división entre las ganancias operacionales y los costos operacionales. El signo de $\beta_{7}$ esperado es negativo ya que la mayoría de teorías acerca de la estructura de capital respaldan el hecho de que la empresa, al tener un margen de ganancias más alto refleja solidez, mayor forma de generar fondos propios y un menor riesgo para cualquier tipo de inversionista reduciendo así sus costos.

\subsubsection{Tangibilidad}

Se considera como el ratio entre activos fijos netos y activos totales. Debido a que esta variable se asemeja al valor de liquidación de la firma o como el colateral de la deuda, el signo esperado para $\beta_{8}$ es 
negativo, esto, si se toma en cuenta la teoría de bancarrota que afirma que entre mayor valor de liquidación tengan los activos de las compañías éstas conseguirán un menor valor en el costo de la deuda.

\subsubsection{Qtobin: Valor del mercado a valor en libros}

Siguiendo a Pozzo (2005), se expresa como el valor en libros del total de activos menos el valor en libro de las acciones más el valor del mercado de las acciones dividido entre el valor en libros del total de activos de la firma. Esta variable, ante un aumento, expresa unas mayores oportunidades futuras de crecimiento. Se espera que $\beta_{9}<0$ sea explicado tanto por la trade-off theory y la peking order theory, frente al costo del capital, ya que la primera afirma que a un aumento en las expectativas de crecimiento hará que los accionistas no quieran compartirlas con los tenedores de deuda, y la segunda enuncia que los recursos propios no son suficientes para financiar dicho crecimiento y se necesitará recurrir al endeudamiento; los dos comportamientos conllevan a una disminución en la tasa mínima de remuneración para los accionistas y una menor tasa para la deuda debido a las perspectivas positivas (Camara, 2012; Pozzo, 2005).
En la tabla 2 se presenta algunos estadísticos de las variables utilizadas en el modelo.

\subsection{Modelos a estimar}

De acuerdo a las características de los datos y a los lineamientos planteados por la literatura, se utilizan modelos de paneles dinámicos con rezagos en algunas variables exógenas (Roodman, 2009), la especificación que se utiliza para la estimación es:

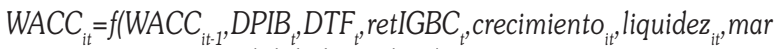
gen_ganancias ${ }_{i t}$, tangibilidadiT, qtobin it $_{\text {J }}$

WACC $_{i t}=\beta_{1}$ WACC $_{i t-1}+\beta_{2} D P I B_{t}+\beta_{3} D T F_{t: 2}+\beta_{4}$ retIGBC $C_{t-1}+\beta_{5}$ crecimiento $_{i t}$ ${ }_{1}+\beta_{6}$ liquidez $_{i t}+\beta_{7}$ margenganancias $_{i t}+\beta_{8}$ tangibilidad $_{i t-1}+\beta_{9}$ qtobin $_{i t}+\mu_{i t}$

La técnica econométrica utilizada en particular la metodología Arellano Bond permite estimar el panel dinámico utilizando la técnica de estimación por diferencias del Método Generalizado de Momentos (GMM), la cual se considera la más apropiada para este tipo de modelos. Esta técnica consiste en transformar las variables independientes por diferencias y estimar el modelo como un sistema de ecuaciones

Tabla 2. Descripción estadística de las variables utilizadas en el modelo

\begin{tabular}{|c|c|c|c|c|c|c|c|c|}
\hline Variable & N & Media & Mediana & Percentil 25 & Percentil 75 & Min. & Max. & $\begin{array}{l}\text { Deviación } \\
\text { Estándar }\end{array}$ \\
\hline WACC & 594 & 7.48 & 7.13 & 5.96 & 8.63 & 0.80 & 23.55 & 2.45 \\
\hline PIB & 782 & 4.85 & 5.10 & 3.90 & 6.20 & 0.20 & 7.70 & 1.92 \\
\hline DTF & 782 & 6.59 & 6.82 & 4.77 & 7.81 & 3.47 & 9.82 & 1.99 \\
\hline Ret_IGBC & 759 & 6.96 & 4.45 & -1.936 & 18.73 & -30.94 & 42.31 & 15.48 \\
\hline Crecimiento Ingresos & 534 & 0.635 & 0.096 & -0.042 & 0.24 & -1.00 & 73.25 & 4.71 \\
\hline Liquidez & 637 & 1.83 & 1.43 & 0.95 & 2.48 & 0.11 & 7.01 & 1.24 \\
\hline Crec_margen_ganancias & 492 & 8.17 & 0.02 & -0.37 & 0.539 & -1.00 & 782.79 & 61.57 \\
\hline Tangibilidad & 646 & 21.60 & 18.36 & 10.97 & 31.90 & 0.00 & 83.96 & 15.54 \\
\hline Q_tobin & 646 & 1.25 & 0.71 & 0.41 & 1.42 & -0.02 & 131.25 & 5.27 \\
\hline
\end{tabular}

Fuente: Elaboración propia. 
conocido como "system GMM" (Arellano \& Bond, 1991).

\section{Resultados}

En la tabla 3 se presenta el reporte para la estimación del modelo dinámico utilizando la metodología propuesta por Arellano Bond para paneles dinámicos (Arellano \& Bond, 1991).

Tabla 3. Estimaciones del modelo mediante la metodología Arellano Bond

\begin{tabular}{|c|c|}
\hline Variable & Modelo \\
\hline WACC $_{t-1}$ & $\begin{array}{l}0.639 * * * \\
(0.0915)\end{array}$ \\
\hline DPIB & $\begin{array}{c}0.115^{\star \star \star} \\
(0.0315)\end{array}$ \\
\hline DTF $_{\mathrm{t}-2}$ & $\begin{array}{c}0.0861^{* * *} \\
(0.0152)\end{array}$ \\
\hline ret_igbc $c_{t-1}$ & $\begin{array}{c}0.00741^{* *} \\
(0.00341)\end{array}$ \\
\hline crecimiento $_{t-1}$ & $\begin{array}{l}-0.0421^{* * *} \\
(0.00925)\end{array}$ \\
\hline liquidez & $\begin{array}{c}0.247^{\star *} \\
(0.114)\end{array}$ \\
\hline margen_ganancias & $\begin{array}{c}-5.00 \mathrm{e}-08^{* \star *} \\
(6.20 \mathrm{e}-09)\end{array}$ \\
\hline tangibilidad $_{\mathrm{t}-1}$ & $\begin{array}{l}-0.00395 \\
(0.00943)\end{array}$ \\
\hline qtobin & $\begin{array}{c}-0.00588^{* *} \\
(0.00238)\end{array}$ \\
\hline Observations & 364 \\
\hline Number of ID & 23 \\
\hline Time Effect & No \\
\hline Test abond AR(1) & $-1.63^{\star}$ \\
\hline Test abond AR(2) & 0,95 \\
\hline Valor $p$ test SARGAN & 0,359 \\
\hline \multicolumn{2}{|c|}{$\begin{array}{l}\text { El error estándar se presenta en paréntesis. }{ }^{* * *} p<0.01,{ }^{* *} p<0.05 \text {, } \\
{ }^{\star} p<0.1\end{array}$} \\
\hline
\end{tabular}

Fuente: Elaboración propia.

De acuerdo con la tabla 3 se puede observar que el modelo estimado tiene los signos acordes a la teoría financiera y resultan estadísticamente significativos, a excepción de la variable tangibilidad.
Las estimaciones realizadas cumplen con los supuestos teóricos asociados a este tipo de modelos, es decir, la estimación es robusta a problemas de heterocedasticidad, el método utilizado se estima considerando los posibles problemas de endogeneidad y los tests de Sargan y Hansen muestra evidencia a favor de la significancia estadística de los instrumentos utilizados.

De acuerdo con la tabla anterior el modelo estimado es:

WACC $_{\text {it }}=0.639$ WACC $_{i t-1}+0.115 \mathrm{DPIB}_{t}+\beta_{2} 0.0861 \mathrm{DTF}_{\mathrm{t}-2}+0.00741$ retIGBC $_{t-1}$ -0.042 crecimiento $_{\text {it } 1}+0.247$ liquide $_{i t}+-5.00^{-08}$ margenganancias $_{i t}-0.0395$ tangibilidad $_{i t-1}-0.00588$ qtobin $^{\text {it }}$

Estos resultados presentan evidencia acerca de la existencia de una inercia en el costo del capital respecto al periodo anterior, así cuando el $W A C C_{i t-1}$ aumenta, se observa que el WACC ${ }_{i t}$ aumenta, este resultado refleja la teoría de la rapidez de ajuste de la estructura del capital (Camara, 2012).

El crecimiento del PIB afecta positiva y contemporáneamente al costo del capital comportamiento acorde con la literatura, siempre y cuando el ciclo económico se encuentre en un periodo expansionista, tal y como ocurre en el periodo estudiado.

Los efectos de la DTF frente a la variable dependiente fueron los esperados, así, cuando esta tasa de captación aumenta en un período su efecto se refleja en el WACC dos períodos después. Esto se puede explicar debido a que los agentes que toman las decisiones al interior de las empresas se ven afectados por las políticas internas de los negocios que evitan que las respuestas a los cambios macroeconómicos sean inmediatas. Es decir, se observa inercia en los costos financieros de las empresas donde no existe rapidez de ajuste frente a variables como la DTF. Se encuentra que los tomadores de decisiones en sus cambios de deuda se toman al menos 6 meses para analizar su tasa de interés.

En cuanto a los factores microeconómicos, la variable de tipo financiero, rendimientos del IGBC en un periodo afectan positivamente el WACC en el siguiente período, comportamiento coherente al modelo CAPM. 
El crecimiento de las ventas en un periodo tiene un efecto negativo sobre el WACC en el siguiente período, este resultado es acorde con la teoría del trade-off que respalda los beneficios que tienen las empresas con perspectivas favorables hacia un fortalecimiento de su participación en los diferentes nichos del mercado.

La liquidez ejerce una influencia contemporánea y positiva sobre el costo del capital, mostrando evidencia de que el costo de capital de las empresas estudiadas castiga el hecho de tener excesos de liquidez lo que implica tener fondos ociosos resultando en un mayor valor del costo de capital. Además otra explicación que se le puede dar a este signo es que de acuerdo con la teoría de los costos de agencia, el mercado valora más las empresas que no tienen excesos de liquidez o en otras palabras capital inutilizado.

La variable margen de ganancias influye contemporáneamente y de forma negativa en el WACC, esto es coherente con la teoría financiera en el sentido de que la empresa al aumentar la generación de fondos propios disminuirá su riesgo.

La tangibilidad afecta contemporáneamente y tiene un efecto negativo sobre el WACC, tal como se presenta en la literatura. Aunque esta variable resulta estadísticamente no significativa es posible que su origen se deba a que las empresas de la BVC pertenecen a sectores muy variados.

Finalmente la variable $\mathrm{Q}$ de Tobin es negativa y significativa, tal como se espera según la teoría de las jerarquías y del trade-off.

Al observar el comportamiento que experimentan las variables explicativas crecimiento y margen de ganancias en el WACC, se deduce que el mercado estará interesado en observar los resultados de las empresas en los periodos pasados para determinar el costo de capital de cada una. Los agentes miran lo que ocurrió en el trimestre anterior para tomar decisiones sobre la capitalización de la empresa.

Los resultados están en línea con estudios similares realizados para la estructura de capital en Latino- américa (Pozzo, 2005) ya que no se encontraron estudios similares para el caso del costo de capital.

\subsection{Análisis de robustez}

Para comprobar la robustez de los resultados del modelo estimado se consideran diferentes especificaciones del modelo. Los resultados de cada uno de los modelos se presentan en la Tabla 4.

- Modelo 1: Utiliza el crecimiento de las márgenes en lugar del margen de ganancias debido a que dada la diversidad de las firmas se pueden presentar distorsiones sobre el WACC por las características particulares de cada sector. Se puede observar que esta estimación conserva las características generales del modelo estimado.

- Modelo 2: utiliza la variable total activos en lugar de la variable tangibilidad, esto debido a que como es sabido el tamaño de las empresas tiene un efecto importante en la estimación del WACC. Como se observa en la tabla 4 la estimación de este modelo conserva las características generales.

- Modelo 3: utiliza la variable endeudamiento en lugar de la variable tangibilidad, debido a que el endeudamiento afecta directamente el WACC. Como se observa en la estimación del modelo los resultados no cambian sustancialmente.

Adicionalmente a los modelos anteriores, para comprobar la robustez de los resultados obtenidos se estimó el promedio anual de cada variable y se corrió un panel para 23 empresas y 10 años. Los resultados de las estimaciones conservan las características generales del modelo estimado inicialmente.

\section{Conclusiones}

Las empresas constituyen la parte central de una economía, su comportamiento afecta directamente el crecimiento del sector y de la industria. Las decisiones sobre los recursos necesarios para la financiación de las operaciones afectan el costo de capital de las empresas y por tanto la gestión que sobre éstos realice el inversionista va a ser fundamental 
para alcanzar un buen desempeño financiero.

Tabla 4. Análisis de robustez

\begin{tabular}{|c|c|c|c|}
\hline Variables & (1) & (2) & (3) \\
\hline WACC $_{t-1}$ & $\begin{array}{l}0.633^{* * *} \\
(0.0921)\end{array}$ & $\begin{array}{c}0.639 * * \star \\
(0.0939)\end{array}$ & $\begin{array}{l}0.634^{\star \star *} \\
(0.0925)\end{array}$ \\
\hline DPIB & $\begin{array}{l}0.146^{\star \star \star} \\
(0.0349)\end{array}$ & $\begin{array}{l}0.132^{\star \star \star} \\
(0.0361)\end{array}$ & $\begin{array}{l}0.143^{\star \star \star} \\
(0.0336)\end{array}$ \\
\hline $\mathrm{DTF}_{\mathrm{t}-2}$ & $\begin{array}{c}0.0945^{\star * *} \\
(0.0170)\end{array}$ & $\begin{array}{c}0.0720 * * * \\
(0.0217)\end{array}$ & $\begin{array}{c}0.0910^{* * *} \\
(0.0167)\end{array}$ \\
\hline ret_igbc $c_{t-1}$ & $\begin{array}{l}0.00892^{* *} \\
(0.00328)\end{array}$ & $\begin{array}{l}0.00750^{* *} \\
(0.00315)\end{array}$ & $\begin{array}{l}0.00887^{\star *} \\
(0.00329)\end{array}$ \\
\hline crecimiento $_{t-1}$ & $\begin{array}{c}-0.0440 * \star \star \\
(0.00814) \\
\end{array}$ & $\begin{array}{c}-0.0465^{\star \star \star} \\
(0.00914)\end{array}$ & $\begin{array}{c}-0.0445^{\star \star \star} \\
(0.00833) \\
\end{array}$ \\
\hline liquidez & $\begin{array}{c}0.298^{\star *} \\
(0.135)\end{array}$ & $\begin{array}{l}0.262^{\star} \\
(0.142) \\
\end{array}$ & $\begin{array}{c}0.292^{\star *} \\
(0.139) \\
\end{array}$ \\
\hline crec_mar & $\begin{array}{c}-0.000618^{* * *} \\
(0.000219)\end{array}$ & $\begin{array}{c}-0.000608^{* * *} \\
(0.000186)\end{array}$ & $\begin{array}{c}-0.000607^{* * *} \\
(0.000214)\end{array}$ \\
\hline qtobin & $\begin{array}{l}-0.00336 \\
(0.00229) \\
\end{array}$ & $\begin{array}{c}-0.0159 * * * \\
(0.00492)\end{array}$ & $\begin{array}{l}-0.00407^{*} \\
(0.00203)\end{array}$ \\
\hline Total Activos & & $\begin{array}{c}-0.265^{\star \star} \\
(0.112)\end{array}$ & \\
\hline endeudamiento & & & $\begin{array}{l}-0.0818 \\
(0.725)\end{array}$ \\
\hline tangibilidad & $\begin{array}{l}-0.00572 \\
(0.0139)\end{array}$ & & \\
\hline Observations & 335 & 335 & 335 \\
\hline Number of ID & 23 & 23 & 23 \\
\hline Test abond AR(1) & $-1,63^{*}$ & $-1.64^{*}$ & $-1.63^{\star}$ \\
\hline Test abond AR(2) & 0.96 & 0.98 & 0.97 \\
\hline $\begin{array}{l}\text { Valor } p \text { test } \\
\text { SARGAN }\end{array}$ & 0.35 & 0.35 & 0.35 \\
\hline
\end{tabular}

Fuente: Elaboración propia.

En las economías es fundamental tener en cuenta los fenómenos asociados a la macroeconomía y a la microeconomía. Al conocer el panorama macroeconómico del país y la situación particular de la firma, el inversionista puede realizar estimaciones de maximización de beneficios y evaluar el retorno de su decisión. Como es sabido, con variables que afecten la economía es de esperar que su efecto se traslade a las empresas.
En este trabajo se establece evidencia de la relación que puede darse entre variables macroeconómicas y microeconómicas sobre el WACC. Para el WACC es fundamental lo que ocurre en la economía y que se puede recoger en las variaciones del producto interno bruto. Los resultados van en línea con la teoría económica sin embargo existe un costo asociado al hecho de presentarse inercia en los costos financieros de las empresas donde no existe rapidez de ajuste de los tomadores de decisiones al requerir al menos 6 meses para adaptarse a las condiciones de deuda del mercado.

El WACC es importante porque constituye la base para la toma de decisiones de inversión en las empresas. En finanzas su estimación está asociada con variables como el costo de la deuda y el costo del capital propio que como observamos no recogen directamente los efectos que variables macroeconómicas y microeconómicas tienen sobre el WACC, sin embargo en esta investigación se identifican y validan las variables macroeconómicas y microeconómicas que influyen en su estimación.

Acorde con la teoría de la rapidez de ajuste de la estructura del capital este estudio presenta evidencia de la inercia en el comportamiento de la variable de interés costo de capital y el costo de capital del trimestre pasado lo que demuestra que las decisiones sobre la financiación de los recursos de las empresas listadas en la BVC dependen de las decisiones pasadas.

Como limitaciones del estudio se tienen la falta de información en algunos periodos y el número de empresas con disponibilidad de datos. Otra limitación importante es que los resultados se establecieron bajo un comportamiento de la economía en un ambiente de crecimiento económico por lo que se esperaría que ante un cambio en el escenario económico las variables estudiadas se comporten de acuerdo con la teoría económica.

Se deja para futuras investigaciones validar el efecto que tendrían las variables estudiadas en el costo de capital de las empresas de la BVC cuando se presenta un ambiente económico contraccionista. 


\section{Referencias}

Ang, J. (1973). Weighted average vs. true cost of capital. Financial Management, 2 (3): 56-60.

Arellano, M. \& Bond, S. (1991). Some tests of specification for panel data: Monte Carlo evidence and an application to employment equations. The Review of Economic Studies, 58: 277-97.

Banco de la República (2012). Informes económicos. Colombia.

Bellalah, M. \& Belhaj, R. (2003). The effect of asymmetric Information on the cost of capital. International Journal of Business, 8 (4): 451-460.

Bokpin, G. (2009). Macroeconomic development and capital structure decisions of firms: evidence from emerging market economies. Studies in Economics and Finance, 26 (2): 129-142.

Camacho, F. \& Meneses, F. (2013). The impact of the price regulation on the cost of capital. Annals of Public and Cooperative Economics, 84 (2): 139-158.

Camara, O. (2012). Capital structure adjustment speed and macroeconomic conditions: U.S MNCs and DCs. International Research Journal of Finance and Economics, 84: 106-120.

Diamond, D. (1989). Reputation acquisition in debt markets. Journal of Political Economy, 97: 828-862.

Easley, D. \& O'hara, M. (2004). Information and the cost of capital. The Journal of Finance, 59 (4): 1553-1583.

Fama, E. \& French, K. (1999).The corporate cost of capital and the return on corporate investment. The Journal of Finance, 54 (6): 1939-1967.

Fuenzalida D. \& Mongrut S. (2010). Estimation of discount rates in Latin America: empirical evidence and challenges. Journal of Economics, Finance and Administrative Science, 15 (28): 7-43

Gama, L. (2007). Costo de capital: estudio de la incidencia de las variables determinantes del costo de capital y el consiguiente reflejo en los estados financieros de las pymes del sector avícola de Bogotá, durante el período 2000-2005. Equidad y Desarrollo, 0(8): 83-94.

Hackbarth, D., Miao, J. \& Morellec, E. (2006). Capital structure, credit risk, and macroeconomic conditions. Journal of Financial Economics, 82 (3): 519-550.

Harris, M. \& Raviv A. (1991). The theory of capital structure. The Journal of Finance, 46 (1): 297-355.

Hirshleifer, D. \& Thakor, A. (1992). Managerial conservatism, project choice and debt. The Review of Financial Studies, 5 (3): 437-470.

Jensen, M. \& Meckling, W. (1976). Theory of the firm: managerial behavior, agency costs and ownership structure. Journal of Financial Economics, 3 (4): 305-360.

Jensen, M. (1986). The takeover controversy: analysis and evidence. Midland Corporate Finance Journal, 4 (2): 1-57.
Korajczyk, R. \& Levy, A. (2003). Capital structure choice: macroeconomic conditions and financial constraints. Journal of Financial Economics, 68 (1): 75-109.

Kraus, A. \& Litzenberger, R. (1973). A state-preference model of optimal financial leverage. The Journal of Finance, 28 (4): 911-922.

Levy, A. (2000). Why does capital structure choice vary with macroeconomic conditions? Trabajo no publicado. New York: Stern School of Business, University,NY.

Levy, A. \& Hennessy, C. (2007). Why does capital structure choice vary with macroeconomic conditions? Journal of Monetary Economics, 54: 1545-1564.

Lewellen, W. (1974). A conceptual reappraisal of cost of capital. Financial Management, 3(4): 63-70.

Merton, R. (1987). A simple model of capital market equilibrium with incomplete information. Journal of Finance, 42: 483-510.

Miller, M. \& Modigliani, F. (1961). Dividend policy, growth and the valuation of shares. Journal of Business, 34 (4): 411-433.

Modigliani, F. \& Miller, M. (1958). The cost of capital, corporation finance and the theory of investment. American Economic Review, 48 (3): 261-297.

Modigliani, F. \& Miller, M. (1963). Corporate income taxes and the cost of capital: a correction. American Economic Review, 53: 433-43.

Myers, S. (1977). Determinants of corporate borrowing. Journal of Financial Economics, 5: 147-175.

Myers, S. \& Majluf, N. (1984). Corporate financing and investment decisions when firms have information that investors do not have. Journal of Financial Economics, 13 (2): 187-221

Pozzo, D. (2005). Análisis de la estructura de capital de las firmas y sus determinantes en América Latina (Tesis de maestría). Universidad Nacional de la Plata, Buenos Aires.

Rivera, J. (2002). Teoría sobre la estructura de capital. Estudios Gerenciales, 18 (84): 31-59.

Roodman, D. (2009). How to do xtabond2: an introduction to difference and system GMM in Stata. Stata Journal, 9 (1): 86-136.

Salazar, S. (2009). Determinantes de la estructura de capital de las empresas colombianas pertenecientes al sector real que cotizaron en la bolsa en el período 1997-2003: un modelo econométrico. Outlier, 0 (5): 1-24.

Stulz, R. (1990). Managerial discretion and optimal financing policies. Journal of Financial Economics, 26: 3-27.

Stulz, R. (2005). The Limits of financial globalization. The Journal of Finance, 60 (4): 1595-1638.

Upadhyay, A. \& Sriram, R. (2011). Board size, corporate information environment and cost of capital. Journal of Business Finance \& Accounting, 38 (9): 1238-1261.

Williamson O. (1988). Corporate finance and corporate governance. The Journal of Finance, 43 (3): 487-511. 